\title{
Body composition, inflammation and thermogenesis in pathways to obesity and the metabolic syndrome: an overview
}

\author{
A. G. Dulloo and J.-P. Montani
}

Department of Medicine, Division of Physiology, University of Fribourg, Fribourg, Switzerland

Address for correspondence: Dr AG Dulloo, Department of Medicine/Physiology, University of Fribourg, Chemin du musée 5, CH-1700

Fribourg, Switzerland.

E-mail: abdul.dulloo@unifr.ch

\begin{abstract}
Summary
According to the World Health Organization, overweight and obesity are defined as abnormal or excessive fat accumulation that presents a risk to health. What constitutes 'abnormal' fat accumulation in this definition is not specified, but this most likely represents a consensus term that encapsulates the importance of adipose tissue dysfunctions, rather than solely excess fat per se, in the pathogenesis of disease entities of the metabolic syndrome, particularly type 2 diabetes and cardiovascular diseases. Over the past decades, such abnormalities in fat accumulation have been linked to a pattern of fat distribution characterized by disproportionate fat deposition in the abdomen; to limits in the capacity of adipose tissue to expand resulting in the burden of fat storage being shifted to 'lean' tissues/organs as ectopic fat; and to the secretion (by both intrinsic and infiltrated cells within the adipose tissue mass) of a plethora of cytokines and other factors which via their autocrine, paracrine and/or endocrine actions underscore a state of chronic low-grade inflammation. These links have formed the basis of a multitude of adipocentric concepts that have stimulated basic and clinical research aimed at explaining differential susceptibilities to cardiometabolic diseases according to diet and lifestyle, birth weight and post-natal growth patterns, menopausal transition and the ageing process, race and ethnicity - often within the framework of hypotheses constructed around thrifty genotypes or thrifty phenotypes, and around overlapping molecular pathways implicated in metabolic inflammation, thermogenesis and body composition regulation. It is against this background of rapidly advancing research in metabolic health - fuelled as much by the search for early markers of cardiometabolic risks as by the search for 'druggable' molecular targets for treating obesity and its comorbidities - that fundamental concepts, controversies and novel research avenues relevant to the theme of 'Body composition, Inflammation and Thermogenesis in Pathways to Obesity and the Metabolic Syndrome' are addressed in this overview and the 10 review articles in this supplement reporting the proceedings of the 6th Fribourg Obesity Research Conference (FORC-2011).
\end{abstract}

Keywords: Adipose tissue, energy expenditure, type 2 diabetes. 
Table 1 Presentation list

\begin{tabular}{|c|c|}
\hline Invited speaker and institution & Authors and title of review articles \\
\hline Manfred Müller & Müller M., Lagerpusch M., Enderle J., Schautz B., Heller M. \& Bosy-Westphal A. \\
\hline Christian-Albrechts University, Kiel, Germany & Beyond the BMI - tracking body composition in pathogenesis of obesity and metabolic syndrome \\
\hline Jonathan Wells & Wells J.C.K. \\
\hline Institute of Child Health, London, UK & $\begin{array}{l}\text { Ethnic variability in adiposity, thrifty phenotypes and cardiometabolic risk: addressing the full range of } \\
\text { ethnicity, including those of mixed ethnicity }\end{array}$ \\
\hline Daniel Konrad & Item F. \& Konrad D. \\
\hline University Children's Hospital, Zurich, CH & Visceral fat and metabolic inflammation: the portal theory revisited \\
\hline Francesca Amati & Amati F. \\
\hline University of Lausanne, $\mathrm{CH}$ & Revisiting the diacylglycerol-induced insulin resistance hypothesis \\
\hline Yves Boirie & Guillet G., Masgrau A., Walrand S. \& Boirie Y. \\
\hline University of Clermont, France & Impaired protein metabolism: interlinks between obesity, insulin resistance and inflammation \\
\hline Zhihong Yang & Yang Z. \& Ming X-F. \\
\hline University of Fribourg, $\mathrm{CH}$ & $\begin{array}{l}\text { mTOR signaling: the molecular interface connecting metabolic stress, aging, and cardiovascular } \\
\text { diseases }\end{array}$ \\
\hline Giovanni Solinas & Solinas $G$. \\
\hline University of Fribourg, $\mathrm{CH}$ & Molecular pathways linking metabolic inflammation and thermogenesis \\
\hline Saverio Cinti & Smorlesi A., Frontini A., Giordano A. \& Cinti S. \\
\hline University of Ancona, Italy & The adipose organ: white-brown adipocyte plasticity and metabolic inflammation \\
\hline Lewis Landsberg & Landsberg L. \\
\hline Northwestern University, Chicago, USA & Core temperature: a forgotten variable in energy expenditure and obesity? \\
\hline Abdul Dulloo & Dulloo A.G., Jacquet J., Montani J-P. \& Schutz Y. \\
\hline University of Fribourg, $\mathrm{CH}$ & $\begin{array}{l}\text { Adaptive thermogenesis in human body weight regulation: more of a concept than a measurable } \\
\text { entity? }\end{array}$ \\
\hline
\end{tabular}

\section{Introduction}

The 6th Fribourg Obesity Research Conference (FORC2011), which was attended by about 100 researchers from both academia and industry based in Switzerland, once again provided a platform for scientists in both basic science and clinical medicine to present and discuss some of the rapidly advancing scientific findings in obesity and metabolic health from a perspective of integrative physiology and nutrition. Its proceedings consist of a total of 10 review articles (Table 1), which, together with abstracts of poster sessions, are published in this supplement. An overview of the topics and main issues addressed at this conference and in the review articles is provided below.

\section{Physical and functional phenotyping}

From a historical perspective, research towards understanding how obesity predisposes to chronic metabolic diseases and the metabolic syndrome has been dominated by concepts centred upon the specific anatomical locations at which fat accumulates in the body. In the 1950s, Vague (1) proposed that excess fat stored on the trunk or android obesity could be metabolically more damaging than fat stored on the limbs (or gynoid obesity). This proposal has been confirmed by a large number of cross-sectional and prospective studies, and it is now recognized that abdominal (or central) obesity is a cardinal feature of the metabolic syndrome, with insulin resistance as a key link between abdominal fat and risks for chronic diseases. Whether specific anatomical compartments in the abdominal region (intra-abdominal, visceral, deep subcutaneous) or other body composition phenotypes (e.g. low muscle mass, ectopic lipid infiltration) confer greater risk for insulin resistance and its complications is however uncertain (2). As emphasized by Müller et al. in this supplement, the tracking of body composition in the pathogenesis of obesity and the metabolic syndrome has to take into account the diversity of body composition phenotypes even within subgroups of obese individuals such as those classified as 'metabolically healthy' and 'metabolically abnormal', as well as within normal-weight individuals classified as 'thin-outside fat-inside' or 'sarcopenic obese' characterized by a high fat mass and low muscle mass. The arguments are put forward that, faced with the results of dynamic phenotyping (by under- and overfeeding) in lean healthy men showing large inter-individual variability in 'metabolic flexibility' (i.e. changes in insulin sensitivity related to insulin secretion) and ' $\beta$-cell plasticity' (i.e. insulin secretion related to insulin sensitivity), it may be necessary to define a metabolically healthy vs. a pathological state by dynamic rather than static phenotyping using both physical (body 
composition) and functional (metabolic, endocrinological and immunological) criteria, and that such approaches will also provide a suitable basis for future genomic research.

The issue of diversity in body composition phenotypes and cardiometabolic risks is also analyzed by Wells, who explored how body composition variability, and in particular its life-course development, may contribute to cardiometabolic risk, and how this scenario may be particularly valuable for understanding ethnic variability in cardiometabolic risk. Indeed, minority ethnic groups within industrialized populations in Europe (South Asians), North America (African- and Hispanic Americans, Pima Indians) or in Australasia (Australians Aborigines, New Zealand Samoans) differ profoundly from those of European descents in their risks for chronic diseases. He proposed a metabolic 'load vs. capacity' model of disease, based on the thrifty phenotype hypothesis of developmental programming, whereby a number of components of organ structure and function that scale positively with birth weight track through development into adult life, and may hence underlie the long-term health effects of early life growth variability. In this 'load-capacity' model, the constituents of lean body mass are proposed to contribute to 'metabolic capacity', a generic trait favouring the maintenance of homeostasis, whereas adiposity (i.e. fat mass) is proposed to contribute to 'metabolic load' which at higher levels challenges metabolic homeostasis, elevating cardiometabolic risks. The topic of ethnic variability in cardiometabolic risks is analysed from the perspective of ecological and evolutionary origins in body composition and metabolism, including adaptation of body size and physique to climate and thermal environment (heat stress vs. cold stress), shortand long-term food availability (quantity and quality), as well as adaptation to infections - with the contention that ethnic variability in both adipose tissue distribution and cytokine biology may indeed reflect differential exposure to infectious diseases.

\section{Portal theory vs. ectopic fat theory}

There are numerous studies, albeit in population groups of European or South Indian ancestries, that do in fact support the concept of a specific role for intra-abdominal fat accumulation (often referred to as visceral obesity) in the link between abdominal obesity and insulin resistance, with the mechanisms attributed to the release of free fatty acids (FFAs) from visceral fat depots, which, by draining into the portal vein, would exert adverse effects on hepatic metabolism. There is, however, no clear proof of such a causal link between visceral adiposity and insulin resistance. Both are in fact common correlates of subcutaneous abdominal adipose tissue accumulation, which, on the basis of its considerably larger mass than visceral fat, could have a greater potential to contribute to insulin resistance through the release of FFAs into the systemic circulation (3). While the issues of regional adipose tissue in the pathophysiology of obesity continue to be a subject for debate, the turn of the 21 st century has been marked by a shift in the attractiveness of the 'portal theory' in favour of the 'ectopic fat theory' which proposes that an important causative link between excess fat and the metabolic syndrome resides in the excessive accumulation of lipids outside 'classical' adipose tissue depots, i.e. in or around organs and tissues that constitute the lean body mass (skeletal muscle, heart, liver, pancreas and kidneys). Indeed, small intracellular reserve of lipids for essential 'housekeeping' functions in non-adipocytes - such as for the maintenance of membrane structure, fluidity and intracellular signalling - is tightly regulated, and if overloaded would lead to cell dysfunction (lipotoxicity) and lipid-induced programmed cell death (lipoapoptosis) (4).

These two theories, however, are not mutually exclusive. As discussed by Item and Konrad in their revisit to the portal theory, the role of ectopic fat storage in the development of insulin resistance and cardiometabolic risks is in accordance with the portal theory because a low storage capacity of portally drained adipose tissue may oversupply the liver with lipids, resulting in ectopic fat accumulation. They emphasized that because intra-abdominal adipose tissue comprises different fat depots and that not all of these depots surround internal (visceral) organs or are drained by the portal vein, their distinct anatomic and functional properties pertaining to lipogenesis, lipolysis and secretion of adipokines and pro-inflammatory cytokines should be kept in mind when analysing function and metabolism of different intra-abdominal depots. The overall conclusion is that although the relative contribution of FFA from visceral fat to the systemic circulation is low, the direct exposure of the liver to increasing amounts of FFAs and/or pro-inflammatory factors released from visceral adipose tissue directly into the portal vein contribute importantly to the plausibility of the portal theory for explaining the development of hepatic insulin resistance, steatosis and inflammation.

\section{Mechanisms of ectopic lipotoxicity}

How ectopic fat storage leads to lipotoxicity and organ dysfunction continues to be an area of intense investigations. Lipotoxicity is believed to occur when fatty acid in excess of the oxidative needs spillover into harmful pathways of non-oxidative metabolism, these being linked to the accumulation of metabolically active lipid intermediates, e.g. diacylglycerols (DAG), ceramides, long-chain fatty acyl-CoAs and acylcarnitines. DAG in particular has been shown to inhibit the insulin signalling cascade in several cell systems and animal models, but its role in the mechanisms by which lipid overload leads to insulin resist- 
ance in humans is equivocal. Following a critical review of the evidence for and against a role of DAG in the development of human muscle insulin resistance, Amati emphasized the need for assessing DAG beyond 'total DAG' as DAG exists as distinct molecular species and stereoisoforms varying in chain lengths and degree of saturation and are located in different cellular compartments (cytoplasmic membrane, organelle membranes and lipid droplets). Consequently, the heterogeneity and dynamic nature of DAG need to be explored in order to identify which DAG moiety and which subcellular localization of DAG subpopulations is (and is not) involved in the pathogenesis of muscle insulin resistance and impaired glucose homeostasis.

The ectopic fat storage theory has also been advanced to explain the mechanisms by which obesity may lead to impaired protein synthesis and muscle functions. In addressing this topic, Guillet et al. first reviewed the literature about the consequences of excessive adipose tissue and their secretions (FFAs, pro-inflammatory cytokines) on skeletal muscle fibre types and contractility, and discussed how changes in muscle typology could impact upon its metabolic capacity in obesity, such as lower oxidative capacity associated with decreased lipid oxidation, thereby favouring an increase in fat storage. They subsequently integrated emerging evidence in support of the notion that as obesity progresses to a state when adipose tissue expandibility reaches its limits and the burden of fat storage is shifted to other organs/tissues, the resulting lipid infiltration in skeletal muscle, together with the secretion of proinflammatory cytokines from the expanded adipose mass, leads to insulin resistance and metabolic inflammation which impair not only glucose and lipid metabolism but also protein synthesis, thereby contributing to the clinical manifestation of sarcopenia, particularly in the elderly.

Obesity and ageing are also independent risk factors for cardiovascular diseases, but there is growing evidence that obesity and ectopic fat storage accelerate the ageing process and exacerbate age-associated cardiovascular diseases. In their analysis of molecular mechanisms that might be implicated in the pathologies common to obesity, ageing and cardiovascular diseases, Yang and Ming provided a comprehensive review of the evidence suggesting that sustained hyperactive mTOR signalling (as opposed to intermittent signalling) plays a crucial role in connecting metabolic stress, ageing and cardiovascular diseases through the stimulation of oxidative stress and inflammatory responses. A mechanistic molecular model is proposed in which in response to chronic positive energy balance, sustained and persistent mTOR signalling is not only a molecular pathway that contributes to the development of obesity and promotes the ageing process, but one which also constitutes a common molecular pathway through which both obesity and ageing predisposes to metabolic inflammation, sarcopenia and increased cardiometabolic risks.

\section{Metabolic inflammation and thermogenesis}

That obesity predisposes to metabolic inflammation is now well recognized, but whether a state of low-grade inflammation can predispose to obesity is much less clear, amidst a large literature suggesting that cytokines and other inflammatory factors released during sepsis or injury can alter thermogenesis, and emerging evidence in rodents that low-grade inflammation occurs in the hypothalamus and locally induces resistance to leptin and insulin may be early events in the development of obesity (5). In reviewing this topic from a perspective of molecular pathways linking metabolic inflammation and thermogenesis, Solinas first emphasized the intricate bidirectional interactions between the immune system and the sympathetic nervous system (SNS) by underlining (i) the actions that the SNS exerts on white blood cells to control immunity and the ability of leukocytes themselves to produce catecholamines, which, along with cytokines, regulate the intensity of the immune response, and (ii) the actions that the innate immune system exerts on SNS control of thermogenesis and core body temperature through the release of cytokines and other signalling molecules. After a comprehensive review of the molecular pathways implicated in innate immunity that may also be involved in energy balance and weight homeostasis, it is concluded that the complex signalling network recruited during metabolic inflammation exerts a balanced action on the modulation of thermogenesis and energy balance, with some pathways inhibiting thermogenesis (and promoting weight gain), whereas other pathways have opposite actions. Consequently, as the author points out, there could be potential limitations and pitfalls in exploiting inflammatory pathways to treat obesity, and that immunomodulation of metabolic inflammation, rather than an anti-inflammatory intervention aiming at its suppression, may be a more promising strategy to increase thermogenesis for the treatment or prevention of obesity and its associated diseases.

To date, however, the only established molecular effector of thermogenesis - whether in response to sepsis, cold or diet - is the mitochondrial protein UCP1 that uncouples oxidative phosphorylation in brown adipose tissue (BAT), a tissue whose presence and function in adult humans have long been debatable. In recent years, investigations into the potential role of BAT in the control of thermogenesis in adult humans have been revitalized by investigations using positron emission tomography coupled to computed tomography scanning, which have revealed the presence of substantial amount of active BAT in the neck and shoulder region in response to relatively mild cold exposure. Consequently, research towards understanding the molecular pathways involved in its disappearance during growth and how it can be recruited and activated may lead to therapeutic targets. In this context, the fact that brown adi- 
pocytes can be found within white fat depots, and that they are recruited in response to cold, catecholamines and other factors released into the circulation from skeletal muscle or heart during exercise (6), have led to a model of different 'types' of brown adipocytes: classical brown adipocytes, which are derived from the myoblast lineage, and brownlike adipocytes, which are either derived from precursors residing within white adipose tissue or directly transdifferentiated from white adipocytes, the latter being referred to as recruitable 'brite' or 'beige' adipocytes. In this supplement, Smorlesi et al. reviewed the recent advances about the origin of both white and brown adipocytes and the evidence in support of the 'transdifferentiation theory' in which these two cell types with opposing functions and contained in varying proportions in multiple fat depots forming the adipose organ can reciprocally convert to each other under appropriate stimuli in order to better satisfy important physiological needs of the organism, including thermogenesis (during cold exposure), energy storage (in obesity) or energy release (during lactation). They argued that the 'browning' effects of the adipose organ can be useful to combat obesity and the metabolic syndrome not only because brown adipocytes are more 'healthy' than white adipocytes but also because the simple size reduction of white adipocytes that characterizes the first steps of transdifferentiation can be useful in determining how to avoid triggering adipocyte death based on critical size and the consequent development of metabolic inflammation as a result of macrophage infiltration. They highlight the molecules and signalling pathways involved in the switching of adipocyte phenotype and conclude that a better understanding of the molecular mechanisms on the basis of white-brown transdifferentiation can be useful to exploit new therapeutic strategies to combat the increasing incidence of metabolic diseases.

\section{Thrifty metabolic traits}

The resurgence of interest in the biology of BAT, a tissue whose primary function is to produce heat for thermoregulatory thermogenesis, has also revived old questions and debates about the importance of thermal regulation and adaptive thermogenesis in the aetiology of human obesity. As Landsberg reminds us, the issues of whether a low core temperature could constitute a thrifty trait that contributes to metabolic susceptibility to obesity and/or resistance to therapeutic slimming have never been adequately addressed in humans. After underlining the high price that mammals have to pay for the maintenance of homeothermy $(40-50 \%$ of 24-h energy expenditure) and the individual variation around the average set point for core temperature, he reviews the evidence that this central set point can be regulated in the interest of energy conservation, and argues that if a lower basal core temperature or a lower temperature in response to lower energy intake is shown to influence the metabolic economy of the obese, this would constitute a physiological rationale for considering core temperature as a therapeutic target in obesity management.

This brings us to another hotly debated issue in human energy metabolism: how important is regulated heat production (i.e. adaptive thermogenesis) in weight homeostasis and obesity development? Unlike core temperature, which is easily and precisely measured without the needs for adjusting body size and body composition, adaptive thermogenesis is not a measurable entity, but is calculated from the parameters of energy balance and body composition, with each parameter's measurement error accumulating in its calculation. In their critical review on this topic, Dulloo et al. first addressed the question of 'what constitutes adaptive thermogenesis' when examining the impact of altered plane of nutrition on changes in the various compartments of energy expenditure, and then discussed the various limitations that can lead to over- or underestimations in its assessment, including definitional and semantics, technical and methodological, analytical and statistical. Despite all these limitations and issues that blur its potential significance in metabolic susceptibility to obesity, it is undeniable that the concept of adaptive thermogenesis has been (and will continue to be) a major driving force for research into mechanistic links between diet/lifestyle, weight dynamics and disease entities of the metabolic syndrome, and for opening new exciting research avenues towards managing obesity and chronic metabolic diseases.

\section{Conflict of Interest Statement}

The authors declare no conflict of interest.

\section{References}

1. Vague J. The degree of masculine differentiation of obesities: a factor determining predisposition to diabetes, atherosclerosis, gout and uric calculous disease. Am J Clin Nutr 1956; 4: 20-34.

2. Dulloo AG, Jacquet J, Solinas G, Montani JP, Schutz Y. Body composition phenotypes in pathways to obesity and the metabolic syndrome. Int J Obes (Lond) 2010; 34(Suppl. 2): S4-17.

3. Frayn KN. Visceral fat and insulin resistance - causative or correlative. Br J Nutr 2000; 83(Suppl. 1): S71-S77.

4. Unger RH, Zhou YT, Orci L. Regulation of fatty acid homeostasis in cells: novel role of leptin. Proc Natl Acad Sci U S A 1999; 96: $2327-2332$.

5. Thaler JP, Schwartz MW. Inflammation and obesity pathogenesis: the hypothalamus heats up. Endocrinology 2010; 151: 41094115 .

6. Whittle AJ. Searching for ways to switch on brown fat: are we getting warmer? J Mol Endocrinol 2012; 49: R79-87. 